\title{
Signal Integrity Analysis of High Speed Interconnects In PCB Embedded with EBG Structures
}

\author{
M. Sindhadevi ${ }^{\dagger}$, Malathi Kanagasabai*, Henridass Arun* and A. K. Shrivastav** \\ Abstract - This paper brings out a novel method for reducing Near end and Far end Crosstalk using \\ Electromagnetic Band Gap structures (EBG) in High Speed RF transmission lines. This work becomes \\ useful in high speed closely spaced Printed Circuit Board (PCB) traces connected to multi core \\ processors. By using this method, reduction of $-40 \mathrm{~dB}$ in Near-End Crosstalk (NEXT) and $-60 \mathrm{~dB}$ in \\ Far End Crosstalk (FEXT) is achieved. The results are validated through experimental measurements. \\ Time domain analysis is performed to validate the signal integrity property of coupled transmission \\ lines.
}

Keywords: High speed interconnects, Electromagnetic Band Gap Structures (EBG), Near-End Crosstalk (NEXT), Far-End Crosstalk (FEXT), Signal Integrity (SI), Eye Pattern (EP), Time Domain Reflectometry (TDR)

\section{Introduction}

Signal integrity (SI) issues gain importance due to increasing speeds and packing densities. High packing densities require decreasing trace width and trace spacing in printed circuit board (PCBs), connected to Multicore processors. One of the major issues is the crosstalk between traces. The trend in these digital systems is to reduce the power supply voltage levels and to increase the clock frequencies, making the noise propagation and coupling along the boards a critical issue. A vast number of available scientific literature have proposed design methodologies to achieve simultaneous switching noise (SSN) mitigation by means of embedded 2-D or 3-D electromagnetic band gap structures (EBGs). This technique was first introduced [1] and allowed suppression of SSN in multilayer PCBs.

EBG structures have contributed to the growing interest in microwave and MMIC applications because they are simple to manufacture and easy to monolithically integrate with other circuits. More efficient and low-cost 2-D EBGs were introduced [2-4]. In these configurations, the power or ground layers are directly patterned with patches in order to provide stop bands over a broader frequency range. Novel geometries were introduced in [5-8] with the specific goal of simultaneously broadening and lowering the frequency range for the noise isolation and suppression. Recently some attention has been addressed to the

$\dagger$ Corresponding Author: Dept. of Electronics and Communication Engineering, Anna University, Chennai, India.

(sindhadevi@gmail.com)

* Dept. of Electronics and Communication Engineering, Anna University, Chennai, India. (mala@annauniv.edu, henrydhas@gmail.com)

** SAMEER, Center for Electromagnetics, Chennai, India. (draks.chennai@gmail.com)

Received: April 15, 2015; Accepted: August 27, 2015 performance of the signal link paths in the presence of signal reference planes of EBG types $[9,10]$.

Mutual coupling effect is mitigated by using EBG structures [11] by properly identifying the location and determining the dimensions of the unit cell EBG and more than $20 \mathrm{~dB}$ mutual coupling suppression of perpendicular transmission line is observed. For efficient EMI reduction in multilayer PCB, cascaded structure of EBG [12] is implemented. Different types of Defective ground structures [13] are used to reduce the crosstalk level in PCBs. A novel array method of EBG unit cells located partially on power / ground planes is proposed for multilayer PCBs and packages [15].

Most of the modern electronic equipments operate in the frequency range from 0.9 to $6 \mathrm{GHz}$ [20]. Reference [16] is also dealing with the reduction of SSN in the frequency range between 0.9 to $4 \mathrm{GHz}$. Thus analysis and suppression of mutual coupling in this is important. Multilayer structures are used for reducing the mutual coupling instead of using single layer for space constraint.

To improve Signal and power integrity in multilayered $\mathrm{PCB}$, complementary split-ring-resonators are utilized [22]. These structures provide a compact and embeddable geometry formed in the Power bus structure to remove the resonance that hinders the signal transfer from one layer to another.

Crosstalk between traces is reduced by placing Via fences [23], Serpentine Guard traces [24], Serpentine Guard trace with vias and rectangular resonators [25] in between the traces. Defective microstrip lines structures [26] have been used for the reduction of crosstalk. The proposed work does not have any elements in between the traces, instead uses Electromagnetic Band gap in the other layer. The comparison of geometrical parameter and the level of mutual coupling reduction between traces are 
Table 1. Comparison with the existing techniques

\begin{tabular}{|c|c|c|c|c|c|c|}
\hline Reference & Technique adopted & $\begin{array}{l}\text { Thickness of the } \\
\text { substrate }(\mathrm{mm})\end{array}$ & $\begin{array}{l}\text { Width of the } \\
\text { line }(\mathrm{mm})\end{array}$ & $\begin{array}{l}\text { Spacing between } \\
\text { the lines }(\mathrm{mm})\end{array}$ & $\begin{array}{l}\text { Length of the } \\
\text { line (mm) }\end{array}$ & $\begin{array}{l}\text { Mutual coupling } \\
\text { reduction }(\mathrm{mm})\end{array}$ \\
\hline [23] & Via fences & 0.508 & 0.97 & 7.57 & 100 & -30.0 \\
\hline [24] & Serpentine guard trace & 0.2 & 0.35 & 0.9 & 500 & -27.86 \\
\hline$[20]$ & Serpentine guard trace with vias & 0.18 & 0.3 & 0.9 & 101.6 & -27.9 \\
\hline [25] & Rectangular shaped resonators & 1.6 & 3 & 9 & 50 & -29.85 \\
\hline$[26]$ & Defective micro stripline structures & 0.762 & 2 & 1.67 & 10 & -35.0 \\
\hline Our work & Electromagnetic band gap structures & 0.8 & 3 & 0.5 & 200 & -40.0 \\
\hline
\end{tabular}

given in Table 1.

This paper introduces a novel method of placing EBG to reduce NEXT and FEXT in the frequency range of 3-4GHz. This method finds an application in the PCB traces connected to new generation processors working on this frequency range. To reduce the mutual coupling between the traces, two types of EBG structures have been placed in array type configuration. As there are no extra structures in between the lines to reduce coupling noise, this method gives extra freedom to the system level designer to arrange the traces. The conventional method of reducing the mutual coupling between traces is by keeping the separation between parallel traces to be thrice the trace width. This is termed as $3-\mathrm{W}$ rule [14]. In our proposed work the traces were separated by $0.5 \mathrm{~mm}$ which is $1 / 6$ of the width of the trace.

The paper is organized as follows. Section 2 contains Characterization of EBG structures. The proposed crosstalk reduction method using Square and Circular Mushroom EBG structures have been modeled and simulated in Section 3. In Section 4, simulation and the measurement results of the proposed structures are given. Time domain analysis in terms of eye diagram characterization, rise time measurements and Time domain reflectometry of the EBG structures are presented in Section 5. Section 6 concludes the paper.

\section{EBG Structure Characterization}

Electromagnetic band gap (EBG) structures forbid the propagation of electromagnetic waves within a particular band. Each unit lattice of these structures can be roughly modeled by parallel LC resonant circuits, which can be employed to improve the designs of filters, reflectors, amplifiers, and patch antennas. Design equations of a Mushroom type EBG structure [19] are given as follows:

$$
\begin{aligned}
& \text { Resonant Frequency } \quad f o=\frac{1}{2 \pi \sqrt{L C}} \\
& C=\frac{w \varepsilon_{o}\left(1+\varepsilon_{r}\right)}{\pi} \cosh ^{-1} \frac{w+g}{g} \\
& L=2 x 10^{-7} h\left[\ln \left(\frac{2 h}{h}\right)+0.5\left(\frac{2 r}{h}\right)-0.75\right] .
\end{aligned}
$$

The resonant frequency, capacitance and inductance for the mushroom type EBG are given in Eqs. (1) - (3) where the ' $w$ ' is the width of the EBG, ' $\mathrm{g}$ ' is the spacing between EBG, ' $h$ ' is the substrate thickness, ' $r$ ' is the radius of the via and the substrate permittivity is ' $\varepsilon_{\mathrm{r}}$ '. The important parameters for the EBG array are the side/radius of the EBG, the gap between the EBGs, height of the substrate and the radius of the via. $\mathrm{L}$ value can be found out by solving Eq. (1) for $f_{0}=3.5 \mathrm{GHz}$ and assuming $C$ value. Solving Eq. (2) by using $\mathrm{C}$ value and fixing ' $\mathrm{g}$ ' along with a substrate of Known permittivity we can find out ' $w$ ' which corresponds to side/ radius of the EBG structure. By solving Eq. (3) using the obtained $\mathrm{L}$ value and the known substrate height, the radius of the via can be found out.

The geometrical parameters of the EBG structure are: $\mathrm{w}=9.9 \mathrm{~mm}, \mathrm{~g}=0.2 \mathrm{~mm}, \mathrm{r}=0.5 \mathrm{~mm}$ and the substrate thickness $\mathrm{h}=0.8 \mathrm{~mm}$ with the relative permittivity of the substrate is 4.3 at $3.5 \mathrm{GHz}$. The equivalent inductance and capacitance values obtained are $41.3 \mathrm{nH}$ and $0.053 \mathrm{pF}$ respectively.

Mushroom type Square and Circular EBG structures and the method of characterizing these structures are shown in Fig. 1. To apply these EBG structures to actual PCBs, it is necessary to quickly and accurately compute the stop band frequencies of interest in the design stage. First the stop band characterization is done using equivalent circuit method.

Using the Eqs. 1-3 the equivalent circuit is designed and the S-parameters were found out. Fig. 2 shows the band stop characterization for the square and circular mushroom EBG structures.

The EBG structures were characterized by commercial EM software (CST). The unit cell is designed and simulated using Eigen mode solver. The stop band response obtained from the simulation is given in Table 2 .

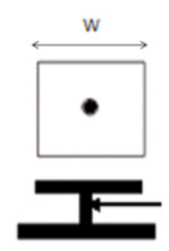

(a)

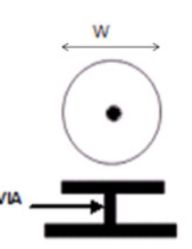

(b)

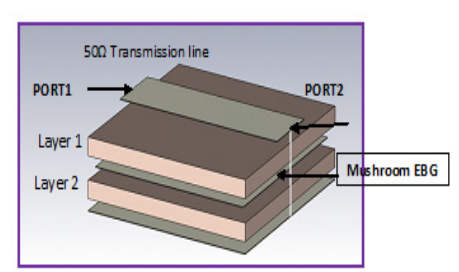

(c)
Fig. 1. Mushroom type EBG structures: (a) Square Circular; (c) Suspended line method for characterizing EBG 


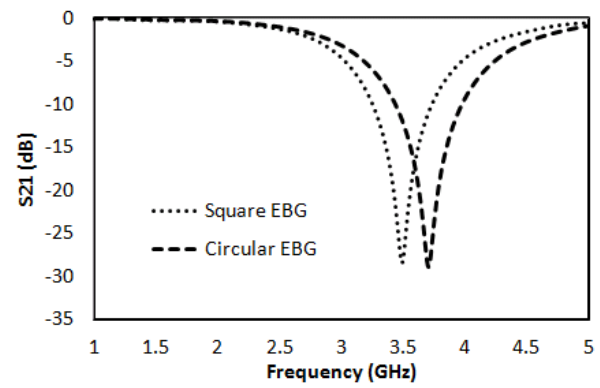

Fig. 2. Transmission coefficient of different Mushroom EBG structures

Table 2. Band Gap By Eigen Mode Solver

\begin{tabular}{c|c|c|c}
\hline Type of EBG & Mode $1(\mathrm{GHz})$ & Mode $2(\mathrm{GHz})$ & Bnad gap $(\mathrm{GHz})$ \\
\hline Square & 2.66 & 4.30 & 1.63 \\
\hline Circular & 2.76 & 3.93 & 1.17 \\
\hline
\end{tabular}

From Table 2 we can find that the stop band frequencies of unit cell Square mushroom EBG is in the frequency range $2.7-4.3 \mathrm{GHz}$ and for Circular mushroom EBG it is $2.8-4.5 \mathrm{GHz}$

\section{Proposed Crosstalk Reduction Method}

\section{Coupled line -}

The density of interconnects on a PCB layer is mainly limited by the crosstalk levels between adjacent interconnects. In order to reduce the magnitude and effect of crosstalk between adjacent interconnects, many design rules and techniques have been widely adopted by high-speed PCB designers. The trace to trace spacing of $3-\mathrm{W}$ rule is mostly followed by high-speed PCB designers.

The main aim of our work is to reduce the spacing between the interconnects from three times the trace width $(3-\mathrm{W})$ to one-sixth $(1 / 6 \mathrm{~W})$ of the trace width so as to achieve compactness in PCB. The proposed structure [18] of two closely spaced transmission lines are shown in Fig. 3. The dimensions of the PCB are of Length ' $L$ ' $=200 \mathrm{~mm}$ and Width ' $\mathrm{W}$ ' $=50 \mathrm{~mm}$. The traces in the $\mathrm{PCB}$ are of $200 \mathrm{~mm}$ and the width ' $\mathrm{W}_{\mathrm{t}}$ ' of $2.99 \mathrm{~mm}$ for trace impedance of $50 \Omega$. The spacing between the lines ' $\mathrm{S}$ ' is $0.5 \mathrm{~mm}(1 / 6$ W). The thickness of the line is $35 \mu \mathrm{m}$. The substrate material is FR4 with relative permittivity of 4.3 and loss tangent 0.0023 .

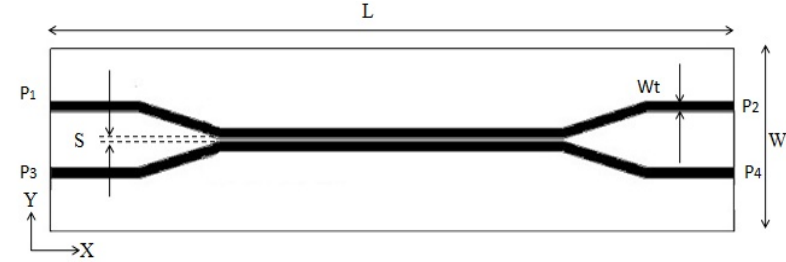

Fig. 3. PCB with the source and victim traces with port numbers

\subsection{Method 1. Square Mushroom EBG}

The analyses of crosstalk reduction were done by embedding the EBG structures in array type configuration. The behavior of the structure is probed at 4 points by means of discrete ports as shown in Fig. 3 and simulation is done by using CST Microwave Studio solver. The Sparameters namely, $\left|\mathrm{S}_{31}\right|$-Near End Crosstalk and $\left|\mathrm{S}_{41}\right|$-Far End Crosstalk were computed and with the coupled microstrip lines placed at a spacing of $0.5 \mathrm{~mm}$.

Fig. 4 shows the proposed three layer structure. On the top of the Layer 1, EBG structures of mushroom types discussed above are placed partially and microstrip transmission lines are placed on Layer 2. In the square configuration the NEXT and FEXT for different iterations are given in Fig. 5 (a) and (b) respectively.

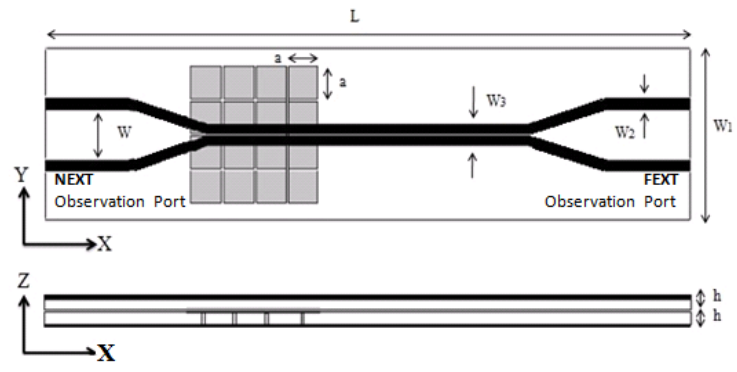

Fig. 4. Proposed three layer PCB structure with Mushroom type square EBG

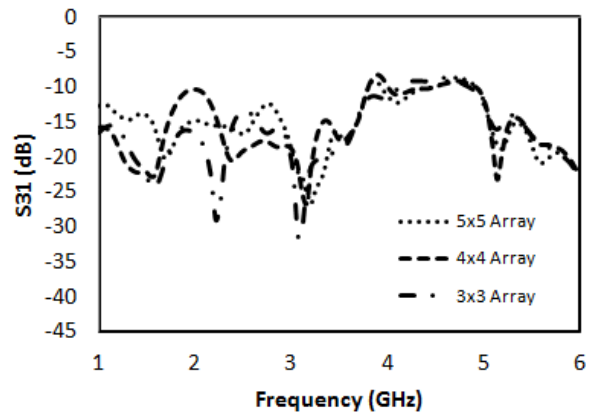

(a)

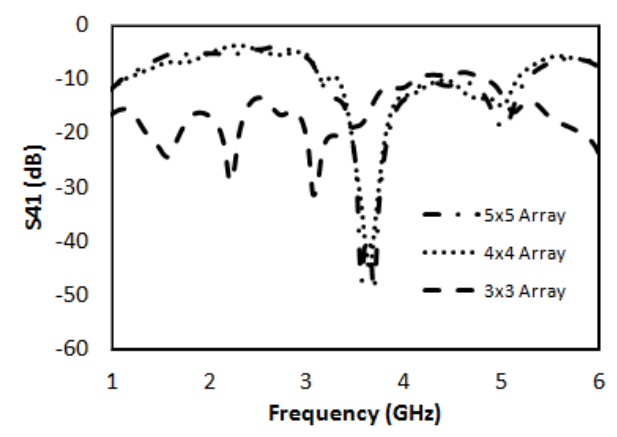

(b)

Fig. 5. S-parametersVs frequency: (a) $\left|\mathrm{S}_{31}\right|$; (b) $\left|\mathrm{S}_{41}\right|$ 
The band of interest is 3.1 to $3.9 \mathrm{GHz}$ used in high speed PCB traces connected to Multi core processors. When the configuration is $3 \times 3$, the reduction in NEXT and FEXT are not achieved. Hence, the array size have been increased and the reduction of crosstalk has been observed for the $4 \times 4$ and $5 \times 5$ arrays. Hence $4 \times 4$ array type was chosen to reduce fabrication complexity.

\subsection{Method 2: Circular Mushroom EBG}

The proposed structure is given in Fig. 6. The analysis was performed by increasing the number of EBGs and corresponding responses were observed.

These NEXT and FEXT values are shown in Fig. 7(a) and (b) respectively. For the $2 \times 2$ and $2 \times 3$ arrays, a gradual reduction in the crosstalk is achieved. And when it is $2 \times 4$

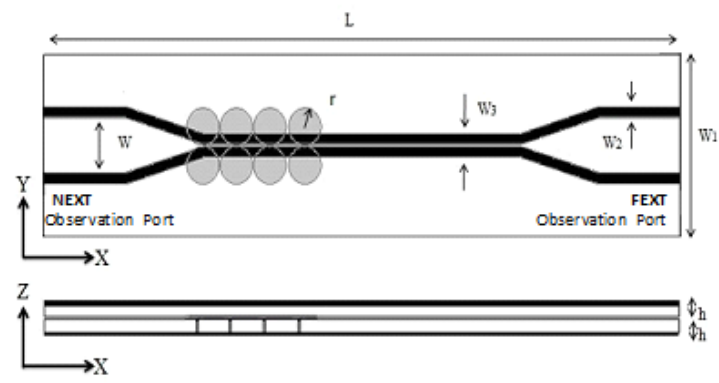

Fig. 6. Proposed three layer PCB structure with Mushroom type circular EBG

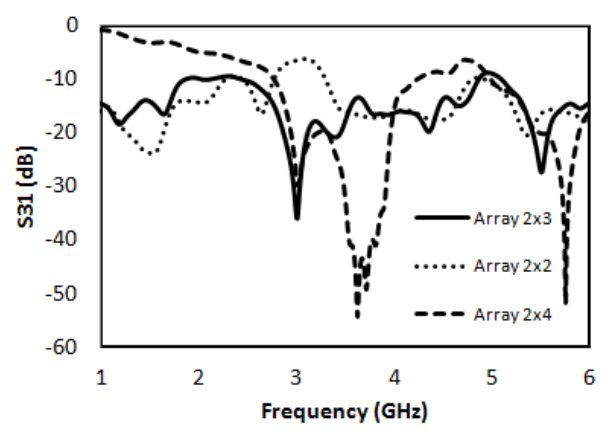

(a)

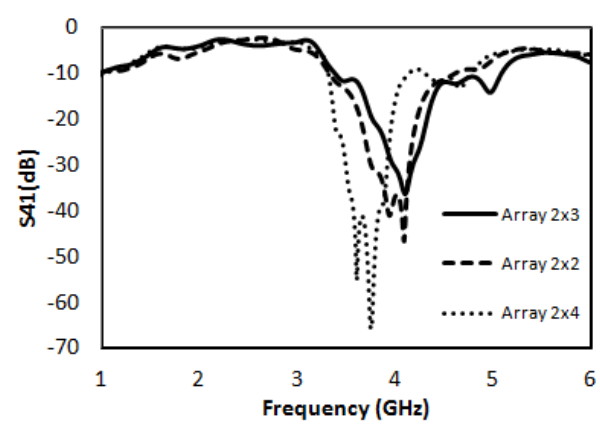

(b)

Fig. 7. Simulated results S-parameters frequency: (a) $\left|S_{31}\right|$ (b) $\left|\mathrm{S}_{41}\right|$ array, good response is achieved for the required band. This response is nearly equal to $4 \times 4$ square EBG responses. So this is selected as another method for reducing crosstalk. The $2 \times 4$ circular EBG configuration reduces the fabrication complexity even more.

\section{Fabrication and Measurement}

The optimized square and circular configuration were fabricated and measured.

\subsection{Square Mushroom EBG}

From the above simulation results the optimum performance is obtained by $4 \times 4$ square mushroom EBG structure. The structure with $4 \times 4$ array of square mushroom EBG is fabricated as shown in Fig. 8. The measured results of the Near End and Far end crosstalk are given in Fig. 9.

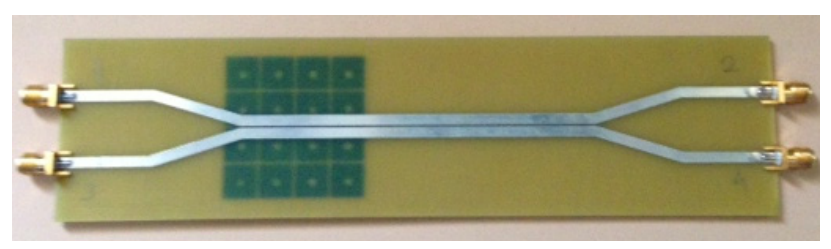

Fig. 8. Fabricated prototype PCB with coupled microstriplines and square mushroom EBG

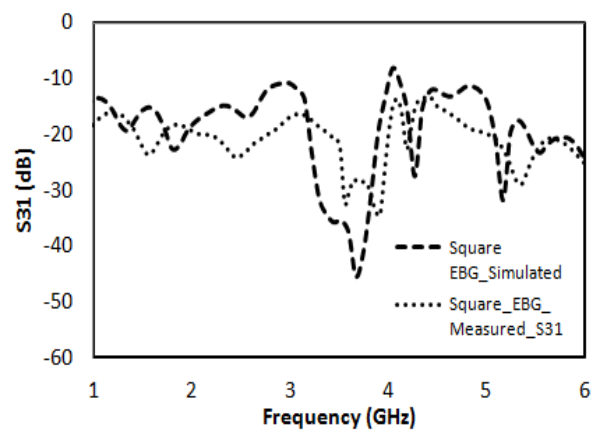

(a)

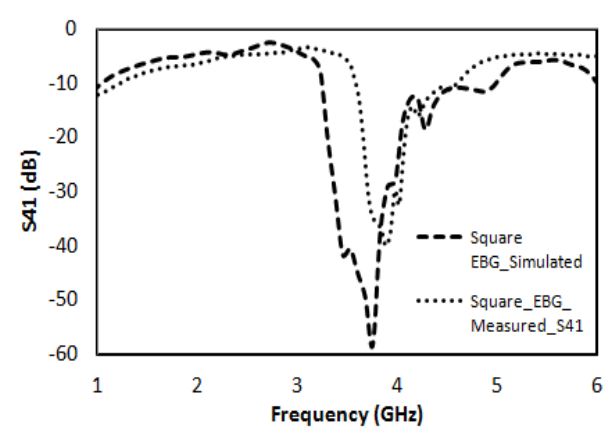

(b)

Fig. 9. Measured results S-parameters Vs frequency: (a) $\left|\mathrm{S}_{31}\right| ;(\mathrm{b})\left|\mathrm{S}_{41}\right|$ 


\subsection{Circular Mushroom EBG}

From the simulated results $2 \times 4$ array gave better performance. The structure with $2 \times 4$ array of circular mushroom EBG is fabricated as shown in Fig. 10.

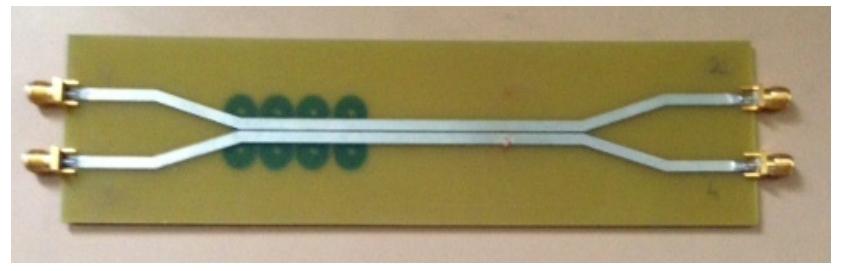

Fig. 10. Fabricated prototype PCB with coupled microstriplines and circular mushroom EBG

The simulated and the measured results of Near End and Far End Crosstalk of circular EBG are given in Fig. 11.

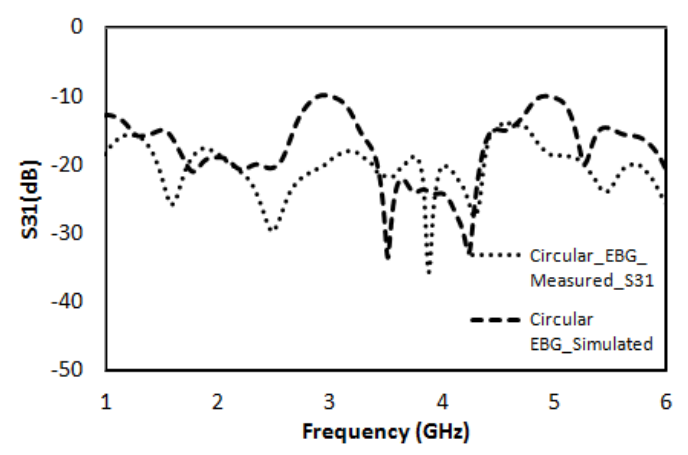

(a)

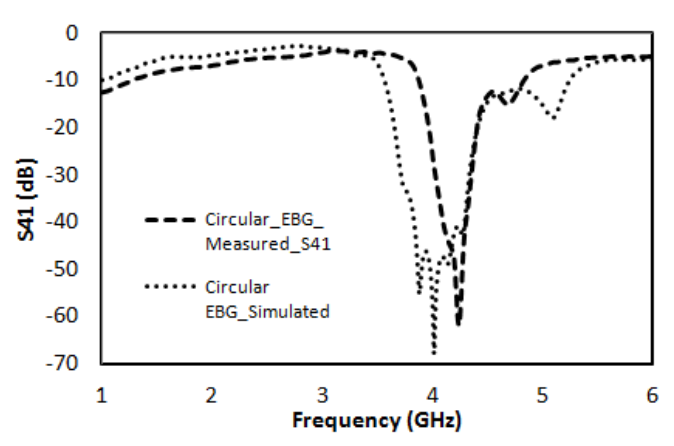

(b)

Fig. 11. Measured results S-parameters Vs frequency: (a) $\left|\mathrm{S}_{31}\right| ;\left(\right.$ b) $\left|\mathrm{S}_{41}\right|$

Table 3. Frequency range of EBG structures for Sparameters below $-15 \mathrm{~dB}$

\begin{tabular}{c|c|c|c|c}
\hline \multirow{2}{*}{ Frequency } & \multicolumn{2}{|c|}{ Square mushroom EBG } & \multicolumn{2}{c}{ Circular mushroom EBG } \\
\cline { 2 - 5 } & $\begin{array}{c}\text { Near end } \\
\text { crosstalk }\end{array}$ & $\begin{array}{c}\text { Far end } \\
\text { crosstalk }\end{array}$ & $\begin{array}{c}\text { Near end } \\
\text { crosstalk }\end{array}$ & $\begin{array}{c}\text { Far end } \\
\text { crosstalk }\end{array}$ \\
\hline $\begin{array}{c}\text { Lower frequency } \\
(\mathrm{GHz})\end{array}$ & 3.23 & 3.42 & 3.00 & 3.37 \\
\hline $\begin{array}{c}\text { Upper frequency } \\
(\mathrm{GHz})\end{array}$ & 4.12 & 4.23 & 4.89 & 4.0 \\
\hline $\begin{array}{c}\text { Bandwidth } \\
(\mathrm{GHz})\end{array}$ & 0.89 & 0.81 & 1.89 & 0.63 \\
\hline
\end{tabular}

The coupling level from Port1 to Port 3 which is the Near end crosstalk and from Port1 to Port4 which is the Far end crosstalk is reduced. The performances of the EBG structures are given in Table 3.

From the above results the crosstalk reduction over a wide band is achieved.

Fig. 12 shows the surface current distribution over the coupled lines for without EBG, with square EBG and Circular EBG at $3 \mathrm{GHz}$. It can be observed that the current distribution over the near end and far end ports are getting reduced with the presence of EBG structures.

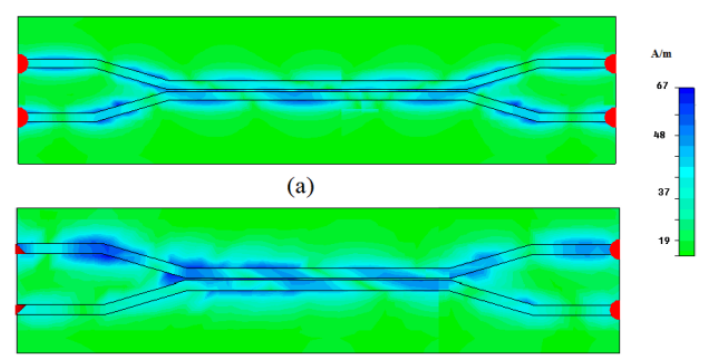

(b)

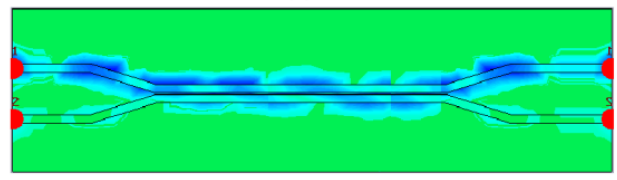

(c)

Fig. 12. Surface Current distribution at $3 \mathrm{GHz}$ : (a) Without EBG; (b) Square EBG; (c) Circular EBG

\section{Time Domain Analysis}

The Signal Integrity analysis explains the signal purity and the jitter associated with the signal. The highest data rate that can be sent through the interconnects either referenced to ground or EBG can be obtained from Signal Integrity analysis. Although the proposed model reduces the NEXT and FEXT levels over a wide frequency range, its effect on propagating a digital signal needs to be analyzed.

Multilayer PCB's disturbs Signal Integrity. Eye diagram analysis is very useful when characterizing a device for a specific digital protocol If the eye is sufficiently open and data transitions don't encroach into a standardized mask, then we can assume that we have compliance to that standard. Hence eye patterns are generated to analyze the Signal Integrity issues when the data lines are referenced over the solid ground plane and EBG plane.

\subsection{Eye diagram characterization}

S-parameters of the structures discussed above are exported from the CST Microwave Studio, 3-D Electromagnetic Solver by Touchstone format. Broad-band models 
are extracted from the simulated S-parameters [16] that are compatible with Advanced design System (ADS) tool.

Eye patterns are generated using ADS circuit simulator by launching a Time-domain Pseudo-Random Bit Sequence Voltage Source. A non return to zero (NRZ) $2^{8}-1$ pattern of pseudorandom binary sequence (PRBS), is given as an input (Port 1). The sequence is coded for different data rates and the signal propagation properties are monitored at the output port (Port 2). The input of the PRBS is coded

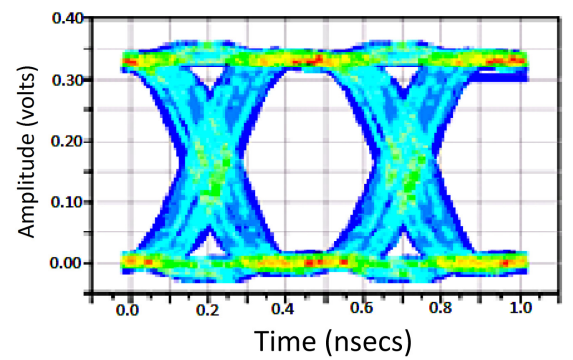

(a)

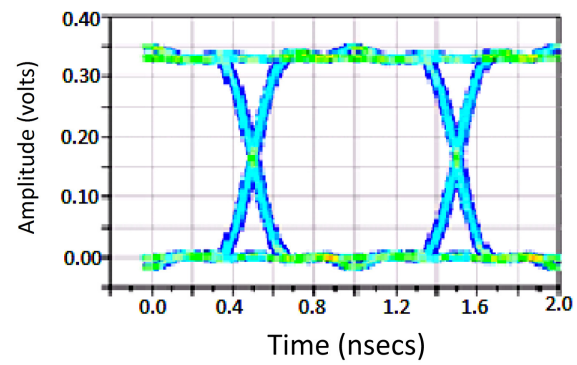

(b)

Fig. 13. Eye diagram of Square type Mushroom EBG: (a) Data rate 2 Gbps; (b) Data rate 1 Gbps.

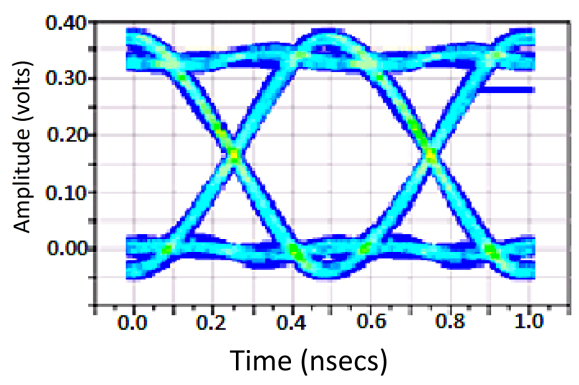

(a)

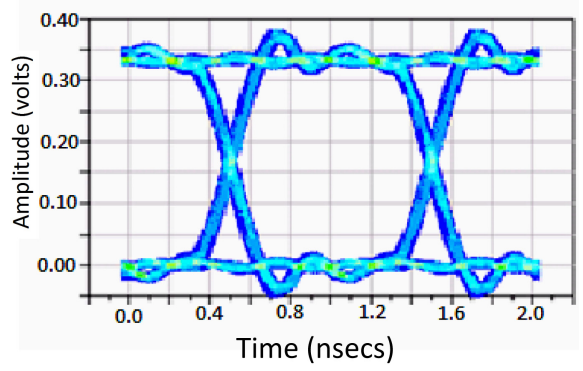

(b)

Fig. 14. Eye diagram of Circular type Mushroom EBG: (a) Data rate 2 Gbps; (b) Data rate $1 \mathrm{Gbps}$ with $0.5 \mathrm{~V}$, voltage swing and rise/fall time of $200 \mathrm{ps}$.

The Two parameters, Maximum Eye Opening (MEO) and Maximum Eye Width (MEW) are used as metrics of the eye pattern quality. Signal to Noise ratio (SNR) and Eye jitter are also measured. Figs. 13 and 14 show the simulated eye diagram of the received signal at the victim line end for square and circular Mushroom EBG respectively. Eye patterns were also generated for a Solid Power/Ground plane of the same dimensions to keep as a reference in estimating the degradation of the signal quality.

The eye pattern parameters of the square and circular EBG are given in Table 4. Maximum Eye opening (MEO) which is the Eye height, Maximum Eye width (MEW), SNR and jitter margin of the two types of EBG are compared with the Solid Power/Ground plane. The degradation of MEO and MEW is $26 \%$ and $9 \%$ for square EBG respectively for a data rate of $2 \mathrm{Gbps}$. The value of degradation is reduced to $0.6 \%$ and $2 \%$ for the data rate of $1 \mathrm{Gbps}$. For circular EBG MEW is good compared with Reference plane for data rate of $2 \mathrm{Gbps}$.

\subsection{Rise time measurement}

A short pulse is given into the traces using Agilent Signal generator 332050A and corresponding NEXT voltage (at port 2) and FEXT voltage (at port 4) levels are measured using Tektronix DPO 4104 Digital Phosphor Oscilloscope.

The measurement setup is shown in Fig. 15 An input square wave, with a rise time of $10 \mathrm{~ns}$ and pulse width of

Table 4. Characteristics of Square and Circular EBG Structures

\begin{tabular}{c|c|c|c|c|c|c}
\hline & \multicolumn{2}{|c|}{$\begin{array}{c}\text { Solid power/ } \\
\text { ground plane }\end{array}$} & \multicolumn{2}{c|}{$\begin{array}{c}\text { With square } \\
\text { EBG }\end{array}$} & \multicolumn{2}{c}{ With circular } \\
\hline $\begin{array}{c}\text { Eye } \\
\text { pattern } \\
\text { parameters }\end{array}$ & $\begin{array}{c}\text { Data } \\
\text { rate }\end{array}$ & $\begin{array}{c}\text { Data } \\
\text { rate } \\
\text { Ebps }\end{array}$ & $\begin{array}{c}\text { Data } \\
\text { rate } \\
2 \mathrm{Gbps}\end{array}$ & $\begin{array}{c}\text { Data } \\
\text { rate } \\
1 \mathrm{Gbps}\end{array}$ & $\begin{array}{c}\text { Data } \\
\text { rate } \\
2 \mathrm{Gbps}\end{array}$ & $\begin{array}{c}\text { Data } \\
\text { rate } \\
1 \mathrm{Gbps}\end{array}$ \\
\hline $\begin{array}{c}\text { Eye height } \\
\text { (Volts) }\end{array}$ & 0.321 & 0.331 & 0.295 & 0.325 & 0.268 & 0.318 \\
\hline $\begin{array}{c}\text { Eye width } \\
\text { (nano } \\
\text { secdonds) }\end{array}$ & 0.45 & 0.99 & 0.36 & 0.97 & 0.5 & 0.97 \\
\hline
\end{tabular}

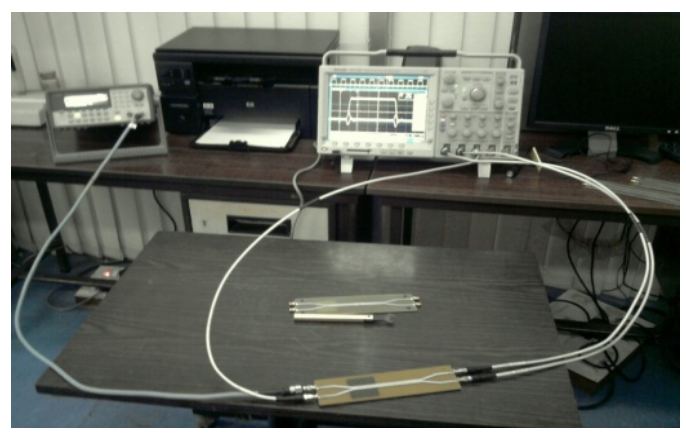

Fig. 15. Experimental Setup for Time Domain Analysis 


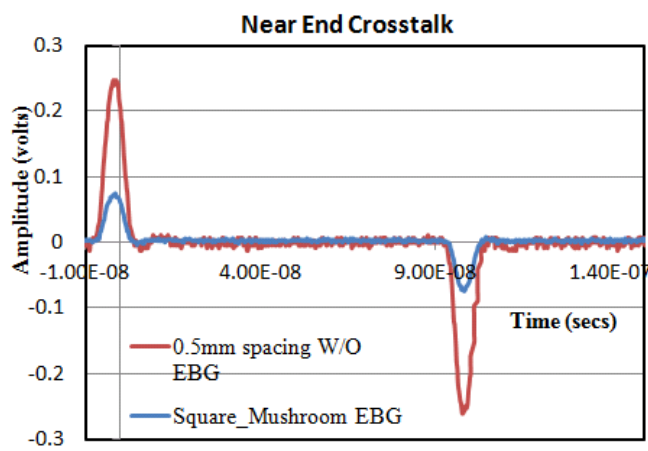

(a)

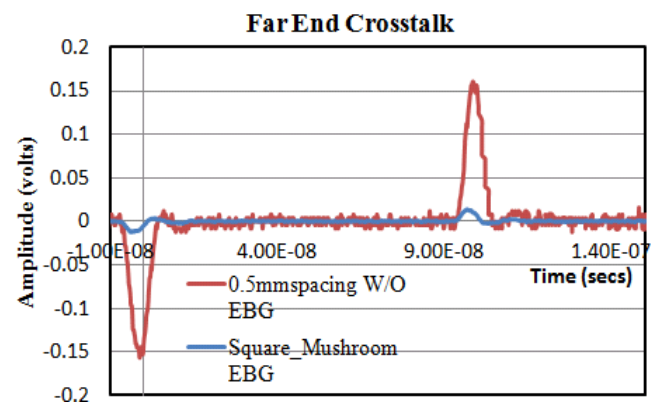

(b)

Fig. 16. Peak Voltage levels of traces with and without square EBG: (a) NEXT; (b) FEXT

$200 \mathrm{~ns}$ with amplitude of $2.5 \mathrm{~V}$ is given at port 2. The NEXT \& FEXT voltage levels are measured with a 4-channel Digital Phosphor oscilloscope at port $1 \&$ port 3 . The peaks in the figures will give the values of crosstalk in the near end and far end.

The results of NEXT and FEXT voltage levels of Mushroom type Square EBG is compared with the structure without EBG and is given in Fig. 16.

The results of NEXT and FEXT voltage levels of traces with mushroom type Circular EBG is compared with the traces without EBG and is given in Fig. 17.

From the results it is observed that the peak voltage level value of NEXT is $0.244 \mathrm{mV}$ without EBG and it is reduced to $0.074 \mathrm{mV}$ when Square and Circular EBG are used. The peak voltage level of FEXT is $-0.156 \mathrm{mV}$ without EBG and it is reduced to $-0.0056 \mathrm{mV}$ for Square EBG and $-0.006 \mathrm{mV}$ for Circular EBG.

\subsection{Time Domain reflectometry (TDR)}

For time domain analysis, Time Domain Reflectometry (TDR) is used [17]. The advantage of performing timedomain measurements is that, the effects of a discontinuities along interconnects on SI can be directly observed, and models of the parasitic elements can be developed for the switching time of interest [17]. The TDR for the PCB trace having Square mushroom EBG, Circular Mushroom EBG and trace without EBG are shown in Fig. 18. From this figure, the impedance of the trace gets increased to

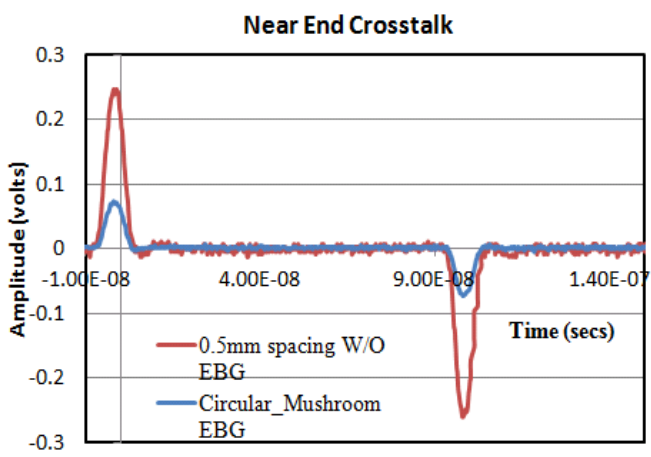

(a)

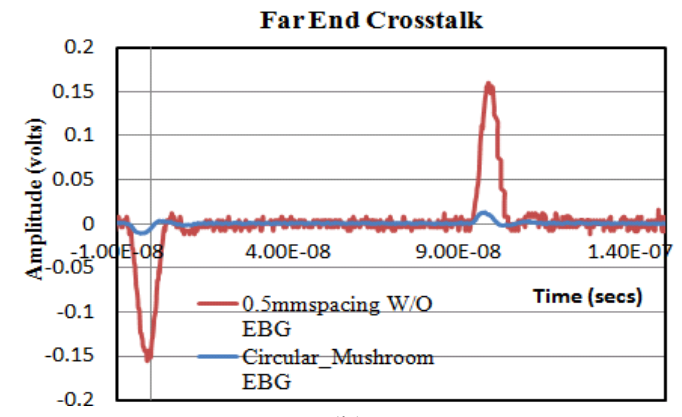

(b)

Fig. 17. Peak Voltage levels of traces with and without circular EBG: (a) NEXT; (b) FEXT

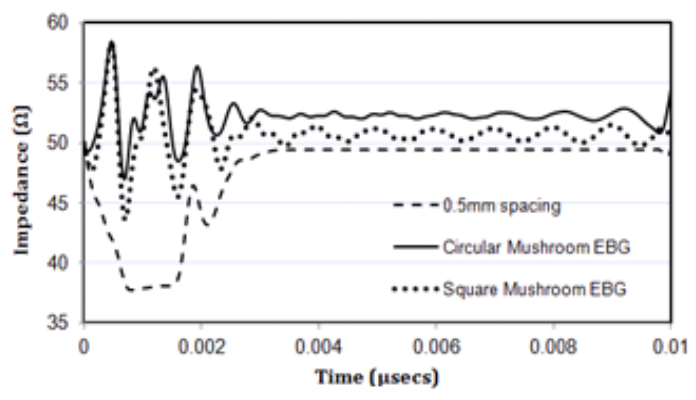

Fig. 18. Impedance Vs Time for traces with different EBG structures

$51.5 \Omega$ and it is near to designed value of $50 \Omega$ due to the parallel high impedance offered by the square mushroom EBG array and the impedance of the trace is $52 \Omega$ for the PCB trace with Circular EBG

\section{Conclusion}

A new method is proposed to reduce NEXT and FEXT level using EBG structures. A $4 \times 4$ array of square Mushroom EBG and 2x4 array of Circular mushroom EBG are designed to reduce the far end crosstalk. The performance of these structures is analyzed in time domain and in frequency domain. Designed structures are fabricated and the measurement agrees with the simulated results. 
From the designed model, circular EBG structure performance is good compared to other structure in terms of impedance matching, crosstalk reduction, and the number of patches used is very less. The coupling level is reduced to a great extent and further studies have to be done on jitter analysis of these structures so that small PCBs with high density of traces can be fabricated.

\section{Acknowledgements}

The authors would like to thank SAMEER CEM, Chennai, India for providing measurement facility.

\section{References}

[1] R. Abhari and G. V. Eleftheriades, "Metallo-dielectric electromagnetic band gap structures for suppression and isolation of parallel-plate noise in high speed circuits," IEEE Trans. Microw. Theory Tech., vol. 51, no. 6, pp. 1629-1639, Jun. 2003.

[2] T. L. Wu, Y. H. Lin, T. K. Wang, and T. Hay, "Electromagnetic band gap power/ground planes for wideband suppression of ground bounce noise and radiated emission in high speed circuits," IEEE Trans. Microw. Theory Tech., vol. 53, no. 5, pp. 2935-2942, Sep. 2005.

[3] T. L. Wu, Y.-H. Lin, and S.-T. Chen, "A novel power planes with low radiation and broadband suppression of ground bounce noise using photonic bandgap structures," IEEE Trans. Microw. Wireless Compon. Lett., vol. 14, no. 7, pp. 337-339, Jul. 2004.

[4] J. Choi, V. Govin, and M. Swaminathan, "A novel electromagnetic bandgap (EBG) structure for mixedsignal system applications," in Proc. IEEE Int. Symp. Radio Wireless Conf., 2004, pp. 243-246.

[5] S. Shahparnia and O. M. Ramahi, "Electromagnetic interference (EMI) and reduction from printed circuit boards (PCB) using electromagnetic bandgap structures," IEEE Trans. Electromagn. Compat., vol. 46, no. 4, pp. 580-587, Nov. 2004.

[6] S. Shahparnia and O. M. Ramahi, "A simple and effective model for electromagnetic bandgap structures embedded in printed circuit boards," IEEE Microw. Wireless Compon. Lett., vol. 15, no. 10, pp. 621-623, Oct.2005

[7] J. Qin and O. M. Ramahi, "Ultra-wideband mitigation of simultaneous switching noise using novel planar electromagnetic bandgap structures," IEEE Microw. Wireless Compon. Lett., vol. 16, no. 9, pp. 487-489, Sep.2006.

[8] T. Kamgaing and O. M. Ramahi, "A novel power plane with integrated simultaneous switching noise mitigation capability using high impedance surface," IEEE Microw.Wireless Compon. Lett., vol. 13, no. 1, pp. 21-23, Jan. 2003.

[9] M.-S. Zhang, Y.-S. Li, C. Jia, and L.-P. Li, "Signal integrity analysis of the traces in electromagneticbandgap structure in high speed printed circuit boards and packages," IEEE Trans. Microw. Theory Tech., vol. 55, no. 5, pp. 1054-1062, May 2007.

[10] T.-K.Wang, T.-W. Han, and T.-L.Wu, "A novel power/ground layer using artificial substrate EBG for simultaneously switching noise suppression," IEEE Trans. Microw. Theory Tech., vol. 56, no. 5, pp. 1164-1171, May 2008.

[11] Cheng-Chi Yu, Meng-Hsiang Haung, Yao-Tien Chang, Luen-Kang Lin, and Tsung-Han Weng, "A Novel Electromagnetic Bandgap (EBG) Structure for Electromagnetic Compatibility (EMC) Application," Progress In Electromagnetics Research Symposium, Beijing, China, March 23-27, 2009.

[12] Hui-Sun He, Xin-Quan Lai, Wen-Dan Xu, Jian-Guo Jang, Ming-Xiang Zang, Qiang Ye, Qiang Wang, "Efficient EMI Reduction in Mulitlayer PCB using Novel Wideband Electromagnetic Band gap structures," International Jouranl of RF an d Microwave Computer Aided Engineering, Vol. 21, No. 4, July 2011.

[13] Henridass A Sindhadevi.M, Karthik N. Alsath, M.G.N, Kumar R.R, Malathi K, "Defective ground plane structure for broadband crosstalk reduction in PCBs," IEEE explore, ICCA-10.1109/ICCCA.2012.6179208, 2012.

[14] Montrose, M.L., EMC and the Printed Circuit Board : Design, Theory and layout Made Simple, IEEE Press, 1998.

[15] Jong Hwa Kwon, Dong Uk Sim, Sang il Kwak, Jong Gwan Yook, "Novel Electromagnetic Bandgap Array Structure On Power Distribution Network For Suppressing Simultaneous Switching Noise And Minimizing Effects On High-Speed", IEEE transactions on Electromagnetic Compatibility, Vol. 52, 365-372, 2010.

[16] Patnam Hanumantha Rao, Madhavan Swaminathan, "A Novel Compact Electromagnetic Bandgap Structure in Power Plane for Wideband Noise Suppression and Low Radiation", IEEE transactions on Electromagnetic Compatibility, Vol. 53, No. 4, 996-1004, 2011.

[17] Spartaco Caniggia, Francescaromana Maradei, "Signal integrity and Radiated emission of high speed digital systems", John Wiley \& Sons, 2008.

[18] Sindhadevi, M, Henridass, A, Malathi, K \& Srivastav, AK 2013, 'Crosstalk Reduction Using Defective Ground Plane Structures in RF Printed Circuit Boards', Arabian Journal of Science and Engineering (AJSE), Vol.39, Issue 2, pp1107-1116, February 2014.

[19] Fan yang and Yahya Rahmat Samii, "Electromagnetic Band gap structures in Antenna Engineering", Cambridge university press, 2009. 
[20] W.-T. Huang C.-H. Lu and D.-B. Lin "Suppression Of Crosstalk Using Serpentine Guard Trace Vias" Progress In Electromagnetics Research, Vol. 109, 3761, 2010.

[21] T. Kamgaing and O. M. Ramahi, "A novel power plane with integrated simultaneous switching noise mitigation capability using high impedance surface," IEEE Microw. Wireless Compon. Lett., vol. 13, no. 1, pp. 21-23, Jan. 2003.

[22] S. Kahng, K. Jang, J. Jeon, H. Oh “A MetamaterialInspired and Embedded Structure to damp the Resoance of the Power/Ground PI planes", Proceedings of EMC Tokyo 2014 (2014 International Symposium on Electromagnetic Compatibility), pp. 109-112.

[23] Asanee Suntives, Arash Khajooeizadeh, Ramesh Abhari. "Using Via Fences for Crosstalk Reduction in PCB Circuits', IEEE proceedings, pp. 34-37, 2006.

[24] K. Lee, H.-B. Lee, H.-K. Jung, J.-Y. Sim, and H.-J. Park, "Serpentine guard trace to reduce far-end crosstalk and even-odd mode velocity mismatch of microstrip lines by more than 40\%," in Electron. Compon. Technol. Conf., Reno, NV, 2007, pp. 329332.

[25] Ding-Bing Lin, Chen-Kuang Wang, Chi-Hao Lu, and Wen-Tzeng Huang, "Using Rectangular shape Resonators to Improve the Far-end Crosstalk of the Coupled Microstrip Lines", PIERS Proceedings, 2011.

[26] Morteza Kazerooni, Mohammad Ali Salari, Ahmad," A Novel Method for Crosstalk Reduction in Coupled Pair Microstrip Lines". International Journal of RF and Microwave Computer-Aided Engineering/Vol. 22, No. 2, March 2012.

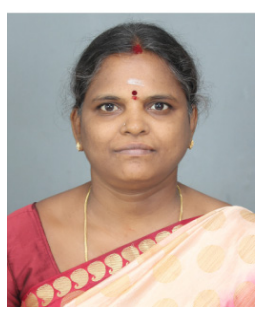

Sindhadevi Muthuramalingam She received B.E. degree in Electronics Engineering from Anna University, Chennai India and Masters degree in Electronics and Communication with specialization in Applied Electronics from College of Engineering, Guindy, Anna University, Chennai, India. She obtained her Doctorate in Information and Communication Engineering from Anna University, India. Her interests include RF and Microwave Engineering, Signal Integrity in High Speed design, Microstrip Patch Antenna design.

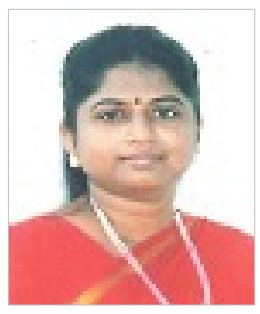

Malathi Kanagasabai She obtained her Ph.D. degree in Microwave Engineering from the Department of Electronics and Communication Engineering, College of Engineering Guindy, Anna University, Chennai. She is currently serving as Associate Professor in the Department of Electronics and Communication Engineering, Anna University, Chennai. Her research interests include microwaves, planar transmission lines, antenna structures, and Signal Integrity Analysis in high speed systems.

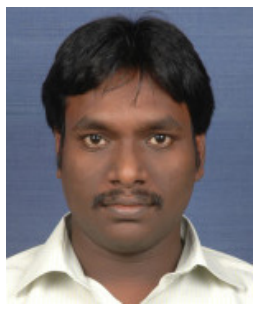

Henridass Arun He received his B.E. degree in Electronics and Communication Engineering from Thiagarajar College of Engineering, and Masters degree in Electronics and Communication with specialization in Communication Systems from College of Engineering, Guindy, Anna University, Chennai, India. He is currently pursuing his Doctorate degree in the Department of Electronics and Communication Engineering, Anna University, Chennai. His research interests include microwaves, MIMO antennas and Signal Integrity Analysis in high speed systems.

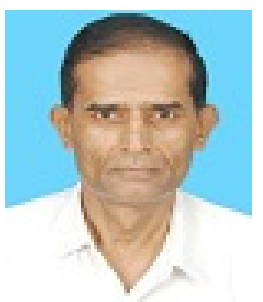

Arun Kumar Shrivastav He completed his $\mathrm{PhD}$ degree from Department of Electronics and Communication Engineering, Anna University, Chennai. He is currently working as scientist $\mathrm{F}$ at Center for ElectroMagnetics, Society for Applied Microwave Electronics Engineering and Research (SAMEER), Chennai, India. His research interests include microwaves, transmission lines, antenna structures, and high speed systems. 